Check for updates

Cite this: Chem. Sci., 2018, 9, 7115

๑ All publication charges for this article have been paid for by the Royal Society of Chemistry

\section{Metal-free alkene oxy- and amino- perfluoroalkylations via carbocation formation by using perfluoro acid anhydrides: unique reactivity between styrenes and perfluoro diacyl peroxides $\uparrow$}

\begin{abstract}
Elena Valverde, ${ }^{a}$ Shintaro Kawamura, (D) ab Daisuke Sekine ${ }^{a}$ and Mikiko Sodeoka (iD *ab
We present a strategy for metal-free, alkene difunctionalization-type, oxy- and amino-perfluoroalkylations, using perfluoro acid anhydrides as practical and user-friendly perfluoroalkyl sources. This method provides efficient access to oxy-perfluoroalkylation products via carbocation formation due to the unique reactivity between styrenes and bis(perfluoroacyl) peroxides generated in situ from perfluoro acid anhydrides. This reaction is also applicable to metal-free intramolecular amino-perfluoroalkylation of styrenes bearing a pendant amino group. Synthetic utility of the oxy-trifluoromethylation products was confirmed by demonstrating derivatization via hydrolysis, elimination, and acid-catalyzed substitution with carbon nucleophiles. The mechanism of the carbocation formation was investigated experimentally and theoretically.
\end{abstract}

Received 10th June 2018

Accepted 20th July 2018

DOI: $10.1039 / \mathrm{c} 8 \mathrm{sc} 02547 \mathrm{a}$

rsc.li/chemical-science

\section{Introduction}

Introduction of perfluoroalkyl groups is an important strategy for modifying the properties of bioactive compounds, agrochemicals and functional materials. ${ }^{1}$ Various methods are available for $\mathrm{C}^{-} \mathrm{CF}_{3}$ bond formation to construct functionalized $\mathrm{CF}_{3}$-containing compounds, and alkene difunctionalizationtype trifluoromethylation has recently attracted particular interest..$^{2-10}$ Styrene derivatives are often used as substrates in these reactions because of their unique reactivity and the utility of the products as $\mathrm{CF}_{3}$-containing synthetic building blocks. ${ }^{2-5}$ For example, transition-metal-catalyzed intermolecular oxytrifluoromethylation to form $\mathrm{C}-\mathrm{O}$ bonds has been well studied (Scheme 1a). ${ }^{3}$ As pioneering works, Szabó ${ }^{3 a}$ and we ${ }^{3 b}$ independently reported Cu-catalyzed intermolecular oxytrifluoromethylation of styrenes with Togni reagent in 2012 . In this reaction, the trifluoromethyl group is introduced into the $\beta$-position, and then 2-iodobenzoate group derived from the Togni reagent is introduced at the benzylic position via carbocation intermediate formation with the aid of copper-catalyst. In contrast to transition-metal-catalyzed reactions, metal-free oxy-trifluoromethylations generally proceed via the following steps: (1) formation of a $\mathrm{CF}_{3}$ radical, (2) formation of an alkyl

${ }^{a}$ Synthetic Organic Chemistry Laboratory, RIKEN Cluster for Pioneering Research, 2-1 Hirosawa, Wako, Saitama 351-0198, Japan. E-mail: sodeoka@riken.jp

${ }^{b}$ RIKEN Center for Sustainable Resource Science, 2-1 Hirosawa, Wako, Saitama 3510198, Japan

† Electronic supplementary information (ESI) available: General experimental procedures, experimental method, compound characterization, and NMR spectroscopic data. See DOI: 10.1039/c8sc02547a radical intermediate by reaction of the $\mathrm{CF}_{3}$ radical and alkene, (3) trapping with an O-radical species. ${ }^{5}$ In 1993, Uneyama reported an electrochemical reaction of butyl acrylate with trifluoroacetic acid (TFA) and $\mathrm{O}_{2}$ as the trapping agent, affording $\mathrm{CF}_{3}$-containing alcohol products. ${ }^{5 b}$ In 2011, Xiao found that $S$ (trifluoromethyl)diphenylsulfonium salt could react with styrenes under aerobic conditions to afford ketone products. ${ }^{5 c} \mathrm{~A}$ similar transformation was also achieved by using $\mathrm{CF}_{3} \mathrm{SO}_{2} \mathrm{Na}$ in the presence of $\mathrm{O}_{2}$, obtaining a mixture of ketone and alcohol products. ${ }^{5 e}$ Lei developed the reaction with $\mathrm{CF}_{3} \mathrm{SO}_{2} \mathrm{Na}$ in the presence of $\mathrm{O}_{2}$ with the aid of $\mathrm{K}_{2} \mathrm{~S}_{2} \mathrm{O}_{8}$ or the combination of $\mathrm{NMP} / \mathrm{PPh}_{3}$, to obtain the ketone or alcohol product selectively. ${ }^{5 ., h} \mathrm{Fu}$ reported oxazoline forming-trifluoromethylation of allylamide with $\mathrm{CF}_{3} \mathrm{SO}_{2} \mathrm{Na}$ by using oxidant. ${ }^{5 g}$ In 2012 , Studer reported an efficient TEMPONa-promoted oxytrifluoromethylation with Togni reagent, ${ }^{5 d, j}$ in which Togni reagent was decomposed via single electron transfer with TEMPONa as an electron donor, affording $\mathrm{CF}_{3}$ and TEMPO radicals and eventually providing the $\mathrm{CF}_{3}$-containing TEMPO adduct by addition to the alkene. In 2015, Tan and Liu reported the metal-free oxy-trifluoromethylation using hydroxamic acids, affording products containing an aminoxyl group. ${ }^{5 i}$ As regards metal-free reaction via a carbocation intermediate, Uneyama developed an electrochemical oxy-trifluoromethylation of butyl methacrylate with TFA and water as the oxygen nucleophile and solvent by careful tuning the current density to oxidize the radical intermediate, obtaining the alcohol product in up to $35 \%$ yield. ${ }^{5 a}$ Further, in 2016, Liu reported an amine-catalyzed intramolecular oxy-trifluoromethylation of alkenes bearing 
(a) Electrophilic oxy-trifluoromethylation transition-metal catalyzed reactions (ref. 3)

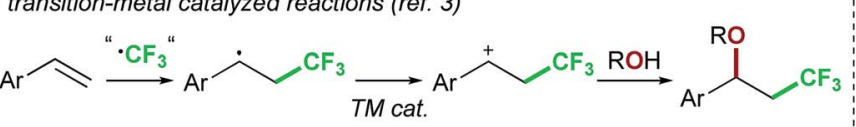

metal-free conditions (ref. 5)

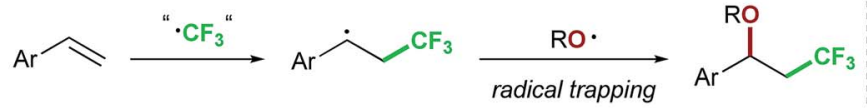

(b) Our previous work of $\mathrm{Cu}$-catalyzed amino-perfluoroalkylation with TFAA/urea $\cdot \mathrm{H}_{2} \mathrm{O}_{2}$ (ref. 9)

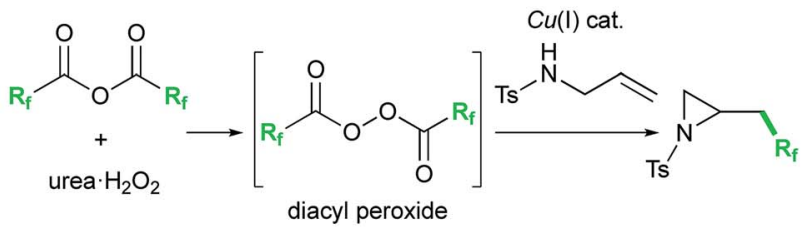

acceleration with $\mathrm{Cu}(\mathrm{l})$ species via SET

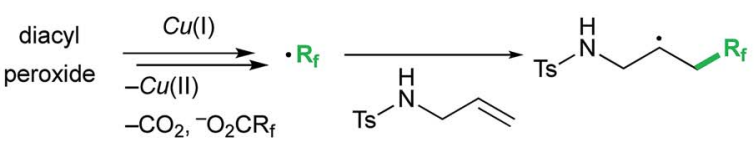

- selectivity control with $\mathrm{Cu}(\mathrm{II})$ species

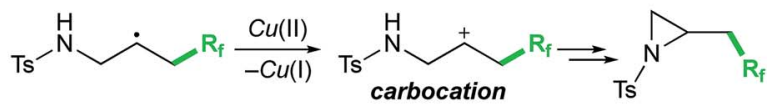

(c) This work

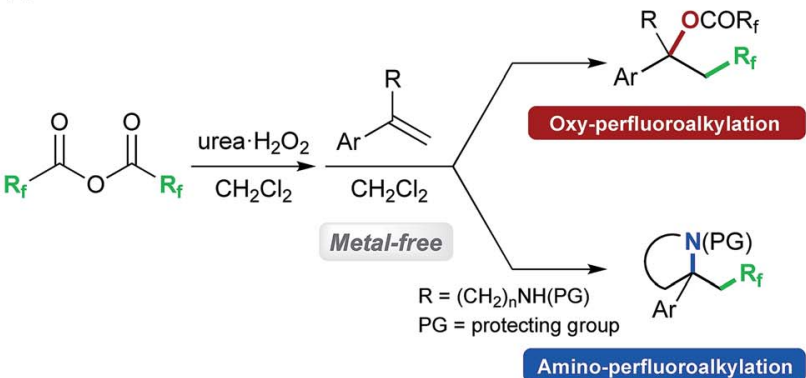

- working hypothesis

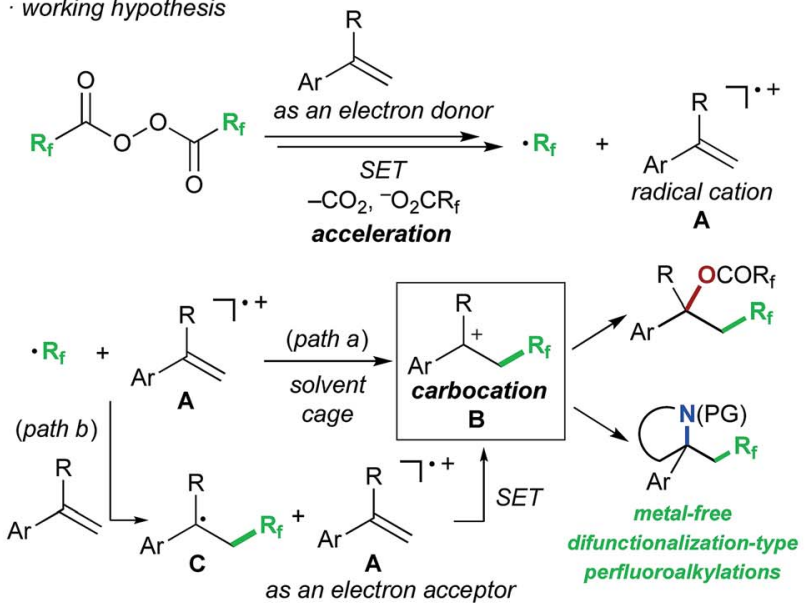

Scheme 1 (a) Proposed mechanisms of previous oxy-trifluoromethylations, (b) our previous work on Cu-catalyzed amino-perfluoroalkylation with TFAA/urea $\cdot \mathrm{H}_{2} \mathrm{O}_{2}$, and (c) metal-free difunctionalization-type perfluoroalkylation of styrenes by using perfluoro acid anhydrides (this work).

a 1,3-diaryl diketone group with Togni reagent, affording dihydrofuran products. ${ }^{5 k, 6}$

Recently, we have been interested in alkene perfluoroalkylation by using perfluoro acid anhydrides, which are convenient and practical perfluoroalkyl sources because of their low cost, ready availability and reasonable stability compared to conventional perfluoroalkylating reagents. ${ }^{9-12}$ We found that perfluoro diacyl peroxides prepared in situ from perfluoro acid anhydrides and urea $\cdot \mathrm{H}_{2} \mathrm{O}_{2}$ showed excellent reactivity and selectivity in allylic perfluoroalkylation ${ }^{9 a}$ and in intramolecular amino-perfluoroalkylation $^{\mathbf{9} \boldsymbol{b}}$ of alkenes in the presence of $\mathrm{Cu}(\mathrm{I})$ salt as a catalyst. Our mechanistic studies of the aminoperfluoroalkylation indicated that it proceeds via (1) formation of a perfluoroalkyl radical $\left(\cdot \mathrm{R}_{\mathrm{f}}\right)$ and $\mathrm{Cu}(\mathrm{II})$ species from the peroxide and $\mathrm{Cu}(\mathrm{I})$ catalyst, (2) addition of the $\mathrm{R}_{\mathrm{f}}$ radical to the double bond of the alkene, (3) oxidation of the resulting alkyl radical with $\mathrm{Cu}$ (II) species to afford a carbocation intermediate with recovery of the $\mathrm{Cu}(\mathrm{I})$ species, and (4) nucleophilic cyclization (Scheme $1 \mathrm{~b}$ ). In the absence of copper catalyst, the reaction of alkenes gave complex mixtures. Exceptionally, reaction of alkenes bearing an aromatic ring at an appropriate position selectively generated perfluoroalkyl group-containing carbocycles, because the aromatic ring acted as a scavenger of the alkyl radical. We were interested in the unique reactivity of styrenes and radical cation species in perfluoroalkylation with the perfluoro acid anhydride/urea $\cdot \mathrm{H}_{2} \mathrm{O}_{2}$ system (Scheme 1c), and postulated that the styrene substrate serves to control the reactivity and selectivity in the formation of the carbocation intermediate without transition-metal-catalyst; ${ }^{9 b}$ i.e., styrene serves as an electron donor to accelerate generation of the perfluoroalkyl radical via decomposition of the diacyl peroxide by SET. Then, addition of the perfluoroalkyl radical to the resulting radical cation $\mathbf{A}$ affords the carbocation $\mathbf{B}$ (path a). Another possibility is that the perfluoroalkyl radical reacts with another styrene molecule (having higher electron density compared to the radical cation $\mathbf{A}$ ), and the resulting benzyl radical intermediate $\mathbf{C}$ is oxidized by the radical cation $\mathbf{A}$ as an electron acceptor to afford the same benzyl cation intermediate B (path b). In this work, we focused on this carbocation formation, as a key process in difunctionalization-type perfluoroalkylation, and aimed to develop metal-free oxy- and amino-perfluoroalkylations of styrene derivatives by using perfluoro acid anhydrides. We also carried out various derivatizations to confirm the synthetic potential of the products.

\section{Results and discussion}

We chose commercially available 4-chlorostyrene 1a as a model substrate to explore the reaction. To our delight, after in situ generation of bis(trifluoroacetyl)peroxide (BTFAP) from trifluoroacetic anhydride (TFAA) with urea $\cdot \mathrm{H}_{2} \mathrm{O}_{2}$ in DCM at $0{ }^{\circ} \mathrm{C}$ for $1 \mathrm{~h}$, reaction with $1 \mathrm{a}$ at $40^{\circ} \mathrm{C}$ for $1 \mathrm{~h}$ afforded the desired oxytrifluoromethylation product $2 \mathbf{a}$. Careful tuning of the ratio of the reagents and the reaction temperature improved the yield. ${ }^{13}$ 
Finally, the reaction with TFAA (10 equiv.) and urea $\cdot \mathrm{H}_{2} \mathrm{O}_{2}(2.5$ equiv.) provided the corresponding oxy-trifluoromethylated product $2 \mathrm{a}$ in $80 \%$ isolated yield (85\% NMR yield) (Scheme 2).${ }^{14}$ The scope of the optimized reaction conditions was then explored using a range of styrene-based substrates (Scheme 3). Various functional groups at the para position were tolerated and the corresponding oxy-trifluoromethylated products were formed efficiently (2a-i) ${ }^{15}$ The usefulness of the reaction was demonstrated in a gram-scale experiment with 4-fluorostyrene $\mathbf{1 b}$, which was transformed into the desired compound $\mathbf{2 b}$ in $93 \%$ yield ( $4.7 \mathrm{~g})$. meta- and ortho-substituted styrene substrates performed well in the oxy-trifluoromethylation reaction $(2 \mathbf{j}-\mathbf{n})$, although higher temperatures were needed for meta-substituted substrate because of slow conversion compared to para- and ortho-substituted styrenes. A disubstituted styrene 10 afforded the target compound 20 in good yield. The generality of the reaction was also assessed with several internal alkenes, which afforded the corresponding difunctionalized products in moderate to good yields (2p-s). Quaternary carbon centres could be constructed successfully, and more complex compounds $2 \mathbf{t}$ and $2 \mathbf{u}$ were isolated in $59 \%$ and $80 \%$ yield, respectively. Finally, this metal-free procedure was applied to the oxy-perfluoroalkylation of styrene-based substrates with other perfluoro acid anhydrides, and the desired products $\mathbf{2} \mathbf{b}^{\prime}$ and $\mathbf{2} \mathbf{d}^{\prime \prime}$ were isolated in excellent yields.

To further explore the ability of the styryl functionality to provide the carbocation intermediate, we next investigated whether pyrrolidines could be obtained by intramolecular amino-perfluoroalkylation of styrene derivatives bearing a pendant amino group via nucleophilic cyclization, based on our previous work. $^{9 b, 16}$ In contrast to metal-free oxyperfluoroalkylation, metal-free amino-perfluoroalkylation has rarely been reported, ${ }^{5 a, 8}$ probably because of the lack of appropriate N-radical trapping agents. Thus, we examined the reaction of styryl group-containing aminoalkene 3a with in situgenerated BTFAP under the optimal conditions for the oxytrifluoromethylation (Scheme 4). ${ }^{17}$ As we had hoped, the amino-trifluoromethylation proceeded well to afford the corresponding $\mathrm{CF}_{3}$-containing pyrrolidine $4 \mathrm{a}$ in $76 \%$ yield. This styrene-driven amino-trifluoromethylation was also applicable to internal alkene $\mathbf{3} \mathbf{b}$, which provided disubstituted pyrrolidine 4b as a syn-diastereomer. ${ }^{18}$ In this reaction, the oxytrifluoromethylation product was obtained as a by-product in $40 \%$ yield, and it was not converted to the aminotrifluoromethylation product $\mathbf{4 b}$ even upon prolonged

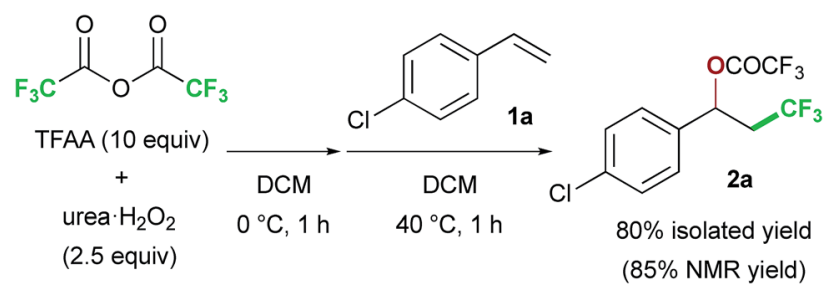

Scheme 2 Metal-free oxy-trifluoromethylation of styrene 1a with TFAA/urea $\cdot \mathrm{H}_{2} \mathrm{O}_{2}$ under optimized conditions.
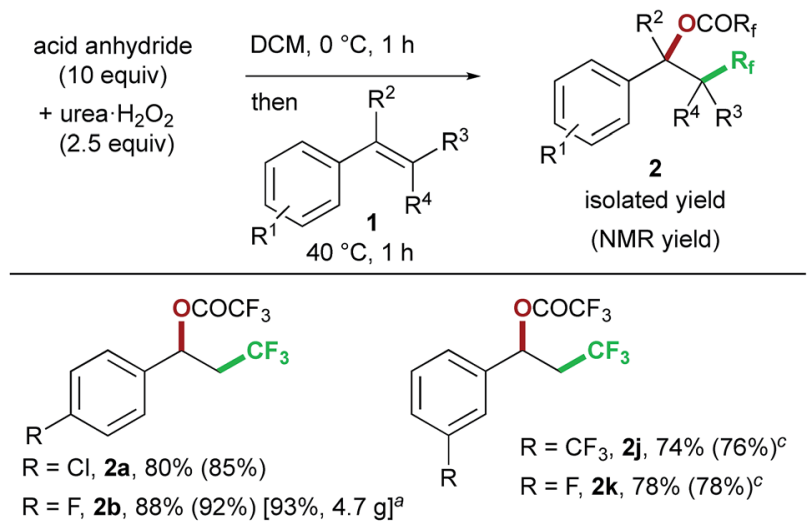

$\mathrm{R}=\mathrm{Br}, 2 \mathrm{c}, 77 \%(79 \%)$
$\mathrm{R}=\mathrm{OCOMe}, 2 \mathrm{~d}$, quant.

$\mathrm{R}=\mathrm{H}, 2 \mathrm{e}$, not determined $(90 \%)$

$\mathrm{R}=\mathrm{Me}, \mathbf{2 f}, 72 \%(76 \%)$

$\mathrm{R}=\mathrm{CH}_{2} \mathrm{Cl}, \mathbf{2 g}, 75 \%(80 \%)$

$\mathrm{R}=t \mathrm{Bu}, \mathbf{2 h}, \mathbf{8 7 \%}(89 \%)$

$\mathrm{R}=\mathrm{OMe}, \mathbf{2 i}, 38 \%(39 \%)^{b}$

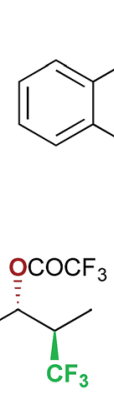

2p, $47 \%(59 \%)$

anti/syn $=2: 1$

$20,75 \%(80 \%)^{c}$

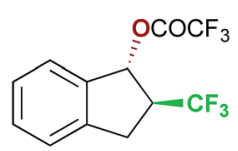

2r, 60\% (61\%)

trans $/$ cis $=8: 1$

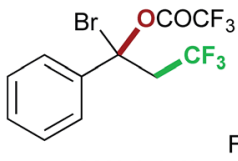

$2 u, 80 \%(79 \%)$

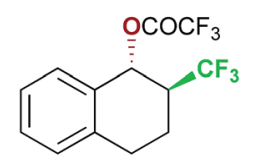

2s, $73 \%(80 \%)$

trans $/$ cis $=2: 1$
$\mathrm{OCOCF}_{3}$<smiles>CCC(C)CC(C)C</smiles>

$\mathrm{R} \mathrm{R}=\mathrm{Cl}, \mathbf{2 1}, 67 \%(70 \%)$

$\mathrm{R}=\mathrm{Br}, \mathbf{2 m}, \mathbf{7 4 \%}(77 \%)$

$\mathrm{R}=\mathrm{Me}, \mathbf{2 n}, 83 \%(85 \%)$

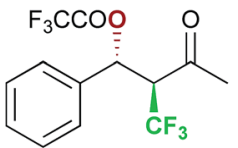

2q, $39 \%(45 \%)^{c}$

anti/syn $=2: 1$<smiles>O=C(OC1(c2ccccc2)CCCC[C@H]1C(F)(F)F)C(F)(F)F</smiles>

2t, 59\% (61\%)

trans $/$ cis $=1: 2$
Scheme 3 Substrate scope of the metal-free oxy-perfluoroalkylation reaction. ${ }^{a}$ Gram-scale synthesis using $2.0 \mathrm{~g}(16 \mathrm{mmol})$ of $1 \mathrm{~b} .{ }^{\mathrm{b}} \mathrm{Run}$ at $0{ }^{\circ} \mathrm{C}$ for 10 min with $\mathrm{Cs}_{2} \mathrm{CO}_{3}$ (5 equiv.) as an additive. ${ }^{\mathrm{C}} \mathrm{Run}$ at $60{ }^{\circ} \mathrm{C}$ in 1,2-dichloroethane.

reaction. This observation suggested that this aminotrifluoromethylation does not proceed via nucleophilic substitution of the oxy-trifluoromethylation product under the conditions. Alkenyl amine $3 \mathbf{c}$ featuring a 6-membered ring as a tethering group in the carbon chain was tolerated, and the spirocyclic product $\mathbf{4 c}$ was formed in high yield. The use of acid anhydrides bearing longer perfluoroalkyl chains furnished $\mathrm{C}_{2} \mathrm{~F}_{5^{-}}$and $\mathrm{C}_{3} \mathrm{~F}_{7}$-substituted pyrrolidines $\mathbf{4 a}^{\prime}$ and $\mathbf{4 a}^{\prime \prime}$ in good yields.

To further expand the chemical space of the perfluoroalkylated compounds, we focused on the reactivity of the perfluoroacetoxy group, as a labile functional group, in the oxytrifluoromethylation products (Scheme 5). First, we examined the reaction of $\mathbf{2 b}$, as a representative substrate, with bases. When DBU was reacted with $\mathbf{2 b}$ in DME, the alcohol $\mathbf{5 b}$ was 


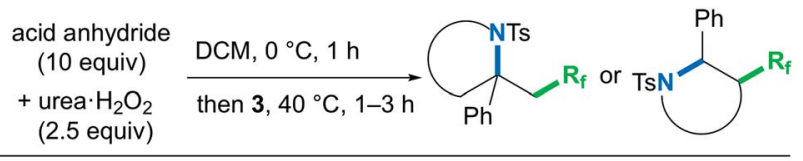

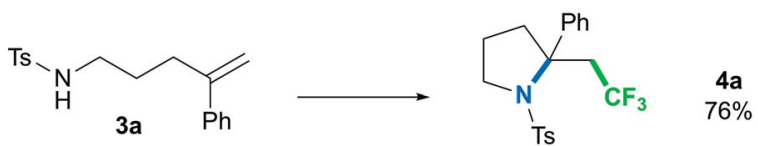<smiles>P/C=C/CCN[AsH]</smiles><smiles>[B-]N1CCC(C(F)(F)F)C1c1ccccc1</smiles>

Ts<smiles>C=C(CC1(CNC)CCCCC1)c1ccccc1</smiles>

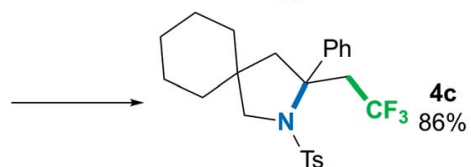<smiles>C=C(CCCNC)c1ccccc1</smiles><smiles>C1CCCCC1</smiles><smiles>[13CH3]N1CCCC1(CC(F)(F)F)c1ccccc1</smiles><smiles>C=C(CCCNC)c1ccccc1</smiles>

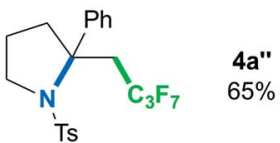

Scheme 4 Metal-free amino-perfluoroalkylation reaction of alkenes.

obtained in quantitative yield. On the other hand, KHMDS as the base was found to give the vinyl product $\mathbf{6 b}$ via elimination reaction. Next, we attempted to construct attractive carbon skeletons and examined $\mathrm{S}_{\mathrm{N}} 1$-type nucleophilic substitution reactions with various carbon nucleophiles in the presence of acid catalysts. The trifluoroacetoxy group was readily dissociated with triflic acid, and trapping of the resulting carbocation with arenes resulted in $\mathrm{C}-\mathrm{C}$ bond formation to give $\mathbf{7 b}$ and $\mathbf{8 b} .^{\mathbf{1 9}}$ In the presence of a catalytic amount of $\mathrm{B}\left(\mathrm{C}_{6} \mathrm{~F}_{5}\right)_{3}$ to promote formation of the carbocation, allylation with allylsilane proceeded to afford $\mathbf{9 b}$ in good yield. ${ }^{20}$ This approach was also applicable to the reaction with a ketene silyl acetal as a nucleophilic partner, providing 10b. The presented procedures for the substitution reactions of the benzylic trifluoroacetoxy group provide rapid access to various perfluoroalkyl-group-containing molecules, which should be useful building blocks in organic synthesis.

Finally, we focused on the reaction mechanism of the perfluoroalkylation (Scheme 1c). The reaction starts with the generation of an electrophilic $\mathrm{CF}_{3}$ radical via decomposition of the diacyl peroxide, i.e. BTFAP which is formed in situ from TFAA and urea $\cdot \mathrm{H}_{2} \mathrm{O}_{2}$. Decomposition of BTFAP would be triggered by SET with styrene as the electron donor and/or by heating (Scheme 6). To trace the decomposition, TEMPO instead of styrene was reacted with in situ-generated BTFAP (Scheme 7a). TEMPO- $\mathrm{CF}_{3}$ adduct 11 was obtained in only $2 \%$ yield, which suggested that thermal decomposition would be very slow under these reaction conditions. ${ }^{21}$ Furthermore, the decomposition could not be observed by ${ }^{19} \mathrm{~F}$ NMR monitoring of

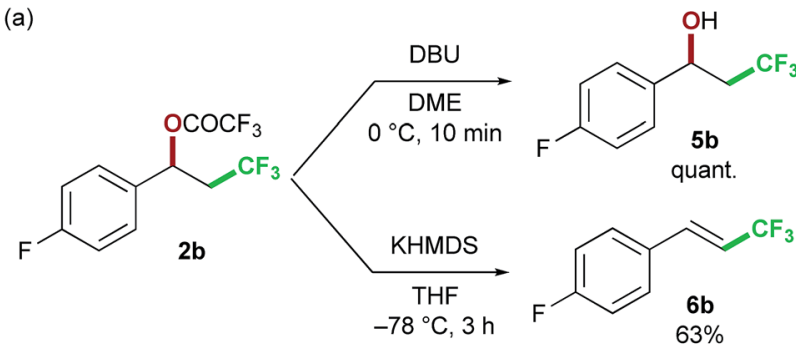

(b)

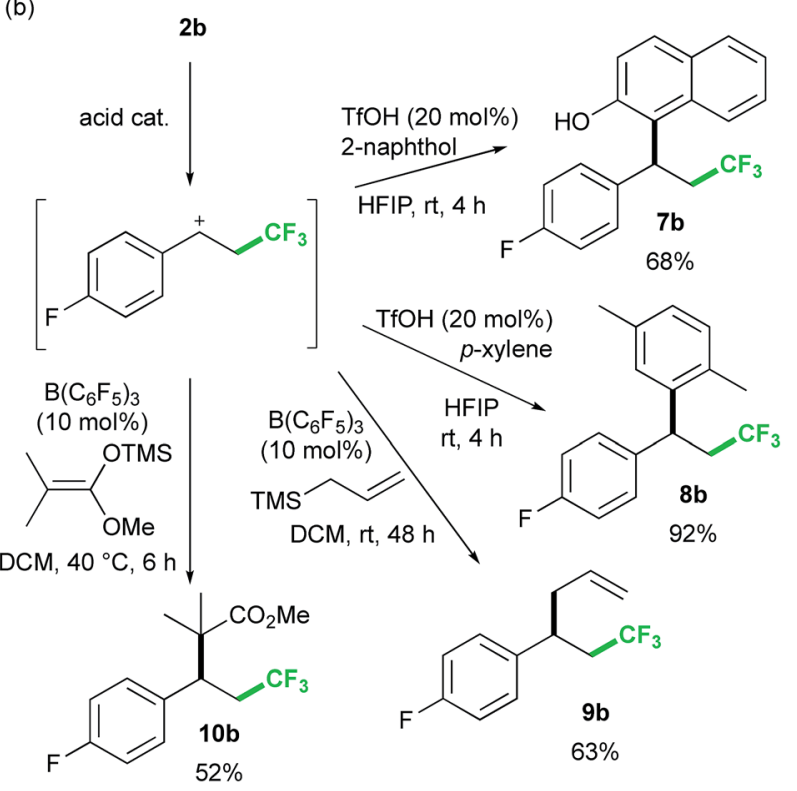

Scheme 5 Examples of derivatization of benzyl trifluoroacetate $2 b$ : (a) hydrolysis and elimination reaction (b) intermolecular $\mathrm{C}-\mathrm{C}$ bond formation reactions.

the peroxide in $\mathrm{CD}_{2} \mathrm{Cl}_{2}$ at $40{ }^{\circ} \mathrm{C}$ without any substrate. ${ }^{13}$ Yoshida similarly found that aromatic compounds such as benzene accelerate the decomposition of BTFAP by SET. ${ }^{11 b}$ The HOMO level of 4-chlorostyrene $1 \mathrm{a}(-6.41 \mathrm{eV})$, used as the model substrate in this work, is higher than that of benzene $(-7.09$ eV). ${ }^{13}$ Accordingly, decomposition of BTFAP was concluded to be induced by SET with styrene under the present conditions at $40{ }^{\circ} \mathrm{C}$, affording $\mathrm{CF}_{3}$ radical and radical cation $\mathrm{A}$. Radical trapping with TEMPO under the optimized conditions afforded the $\mathrm{CF}_{3}$-containing TEMPO-benzyl adduct 12, generated via the benzyl radical intermediate $\mathbf{C}$, in $7 \%$ yield (Scheme $7 \mathrm{~b}$ ). In addition to 12, TEMPO- $\mathrm{CF}_{3} 11$ was formed, together with a mixture of oxy-trifluoromethylation products $2 \mathbf{a}$ and its

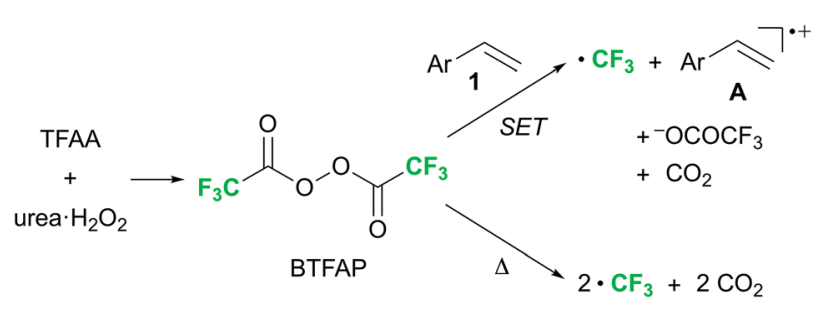

Scheme 6 Possible pathway of decomposition of BTFAP. 
(a)

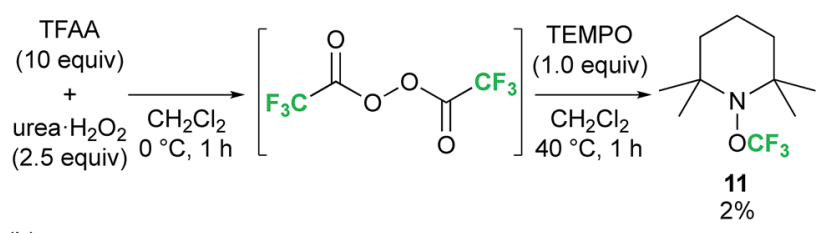

(b)
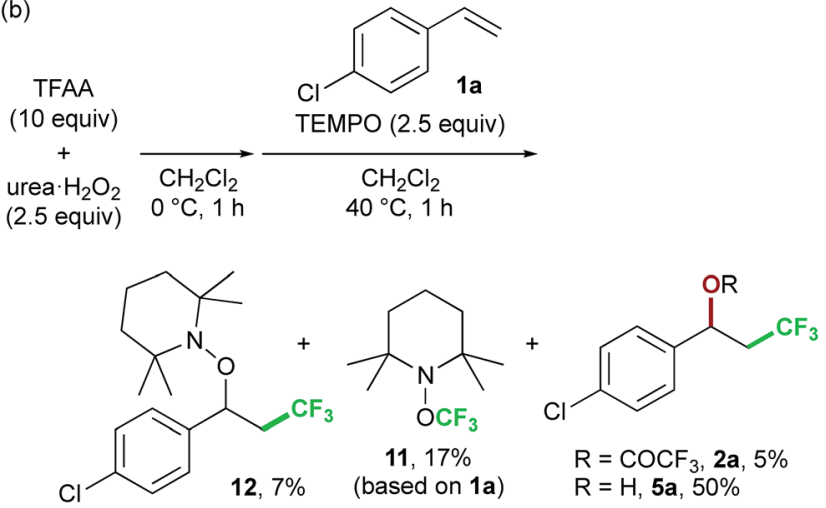

Scheme 7 TEMPO trapping test: (a) reaction of TEMPO with BTFAP and (b) oxy-trifluoromethylation in the presence of TEMPO.

hydrolysis product 5a. Furthermore, a known radical probe alkene 13, 1-phenyl-1-(trans-2-phenylcyclopropyl)ethane, ${ }^{\mathbf{B b}}$ was subjected to the reaction (Scheme 8 ). The corresponding ringopening product $\mathbf{1 4}$ was formed via the radical intermediate as the major product, along with a complex mixture of other products. ${ }^{13}$ These results proved that the $\mathrm{CF}_{3}$ radical reacts with styrene 1a (path b, Scheme 1c), although both path a and path b may be operated. Indeed, DFT calculation indicated that the activation energy of the reaction of the $\mathrm{CF}_{3}$ radical with $1 \mathrm{a}$ is low $\left(\Delta G^{\ddagger}=+10.3 \mathrm{kcal} \mathrm{mol}^{-1}\right.$, Scheme 9a). Next, we considered the oxidation step of the benzyl radical to the carbocation $\mathbf{B}$. Comparison of the calculated LUMO levels of potential oxidants, BTFAP and radical cation $\mathbf{A}$, indicated that radical cation $\mathbf{A}(-6.26 \mathrm{eV})$ has a lower LUMO level than that of BTFAP $(-2.35 \mathrm{eV})$. In addition, the LUMO level of $\mathbf{A}$ was closer to that of $\mathrm{Cu}(\mathrm{II})\left(\mathrm{O}_{2} \mathrm{CCF}_{3}\right)_{2}(-5.09 \mathrm{eV})$ which was reported to oxidize the radical intermediate in the amino-perfluoroalkylation reaction (Scheme 1b). ${ }^{\boldsymbol{g} \boldsymbol{b}}$ Thus, radical cation $\mathbf{A}$ was considered to act as the oxidant, affording carbocation $\mathbf{B}$, which leads to the desired products. The DFT calculated activation energy of oxidation of benzyl radical $\mathbf{C}$ with $\mathbf{A}$ was $\Delta G_{\mathrm{ET}}^{ \pm}=+6.2 \mathrm{kcal} \mathrm{mol}^{-1}$ (Scheme $9 \mathrm{~b}$ ), which is much lower than that of the addition of $\mathrm{CF}_{3}$ radical to 1a suggesting rapid conversion of the highly reactive benzyl radical $\mathbf{C}$ to the metastable benzyl cation intermediate $\mathbf{B}$. These

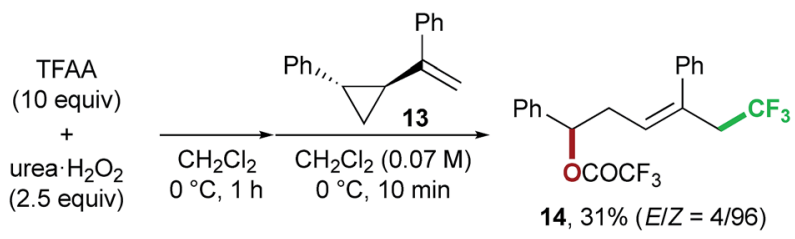

Scheme 8 Radical probe test using 13. (a)<smiles>C=Cc1ccc(Cl)cc1</smiles>

(b)<smiles>C=C[C+](C)C=Cc1ccc(Cl)cc1</smiles><smiles>C=Cc1ccc(Cl)cc1</smiles>

Scheme 9 Activation energies of (a) addition of $\mathrm{CF}_{3}$ radical to 1a and (b) SET between benzyl radical $\mathrm{C}$ and radical cation $\mathrm{A}$.

mechanistic studies supported our original hypothesis shown in Scheme 1c, in which substrate styrene itself acts as SET donor to trigger the perfluoroalkyl radical formation from the diacyl peroxide. The resulting perfluoroalkyl radical could react with styrene affording benzyl radical intermediate $\mathbf{C}$, which is rapidly oxidized to the benzyl cation $\mathbf{B}$ by the radical cation $\mathbf{A}$. The generated benzyl cation intermediate $\mathbf{B}$ is trapped by the perfluoro carboxylate anion or amine yielding the desired oxy- and amino-perfluoroalkylation products, 2 and 4.

\section{Conclusions}

We have developed a mild and efficient method for the metalfree oxy- and amino-perfluoroalkylation of styrenes via carbocation intermediates, using perfluoro acid anhydrides as inexpensive and practical perfluoroalkyl sources. The oxytrifluoromethylation products were derivatized to a variety of $\mathrm{CF}_{3}$-containing unique molecules. We believe this method will prove useful in medicinal and agro-chemistry discovery programs. In addition, the unique reactivity between styrene and perfluoro diacyl peroxide may provide clues to design new reactions and catalysts in the future.

\section{Conflicts of interest}

There are no conflicts to declare.

\section{Acknowledgements}

This work was supported by JSPS KAKENHI (No. 15K17860) and Project Funding from RIKEN. E. V. is grateful for a JSPS Postdoctoral Fellowship (15F15340). The computational study was conducted on the HOKUSAI-GW (RIKEN).

\section{Notes and references}

1 Selected reviews: (a) Y. Zhou, J. Wang, Z. Gu, S. Wang, W. Zhu, J. L. Aceña, V. A. Soloshonok, K. Izawa and H. Liu, Chem. Rev., 2016, 116, 422; (b) J. Wang, M. SánchezRoselló, J. L. Aceña, C. del Pozo, A. E. Sorochinsky, 
S. Fustero, V. A. Soloshonok and H. Liu, Chem. Rev., 2014, 114, 2432; (c) T. Fujiwara and D. O'Hagan, J. Fluorine Chem., 2014, 167, 16.

2 Selected reviews: (a) Y. Tian, S. Chen, Q.-S. Gu, J.-S. Lin and X.-Y. Liu, Tetrahedron Lett., 2018, 59, 203; (b) X. Wang and A. Studer, Acc. Chem. Res., 2017, 50, 1712; (c) A. Prieto, O. Baudoin, D. Bouyssi and N. Monteiro, Chem. Commun., 2016, 52, 869; (d) T. Courant and G. Masson, J. Org. Chem., 2016, 81, 6945; (e) T. Koike and M. Akita, Acc. Chem. Res., 2016, 49, 1937; (f) S.-M. Wang, J.-B. Han, C.-P. Zhang, H.-L. Qin and J.-C. Xiao, Tetrahedron, 2015, 71, 7949; $(g)$ J. Charpentier, N. Früh and A. Togni, Chem. Rev., 2015, 115, 650; $(h)$ C. Alonso, E. M. de Marigorta, G. Rubiales and F. Palacios, Chem. Rev., 2015, 115, 1847; (i) T. Besset, T. Poisson and X. Pannecoucke, Chem.-Eur. J., 2014, 20, 16830; (j) Y. Shimizu and M. Kanai, Tetrahedron Lett., 2014, 55, 3727; (k) S. Barata-Vallejo, B. Lantaño and A. Postigo, Chem.-Eur. J., 2014, 20, 16806; (l) H. Egami and M. Sodeoka, Angew. Chem., Int. Ed., 2014, 53, 8294; $(\mathrm{m})$ E. Merino and C. Nevado, Chem. Soc. Rev., 2014, 43, 6598; (n) J. Xu, X. Liu and Y. Fu, Tetrahedron Lett., 2014, 55, 585; (o) P. Chen and G. Liu, Synthesis, 2013, 45, 2919.

3 Selected reports on transition-metal-catalyzed oxytrifluoromethylations of styrenes: (a) P. G. Janson, I. Ghoneim, N. O. Ilchenko and K. J. Szabó, Org. Lett., 2012, 14, 2882; (b) H. Egami, R. Shimizu and M. Sodeoka, Tetrahedron Lett., 2012, 53, 5503; (c) R. Zhu and S. L. Buchwald, J. Am. Chem. Soc., 2012, 134, 12462; (d) Y. Yasu, T. Koike and M. Akita, Angew. Chem., Int. Ed., 2012, 51, 9567; (e) R. Zhu and S. L. Buchwald, Angew. Chem., Int. Ed., 2013, 52, 12655; $(f)$ A. Deb, S. Manna, A. Modak, T. Patra, S. Maity and D. Maiti, Angew. Chem., Int. Ed., 2013, 52, 9747; $(g)$ X.-Y. Jiang and F.-L. Qing, Angew. Chem., Int. Ed., 2013, 52, 14177; $(h)$ N. O. Ilchenko, P. G. Janson and K. J. Szabó, J. Org. Chem., 2013, 78, 11087; (i) A. Carboni, G. Dagousset, E. Magnier and G. Masson, Org. Lett., 2014, 16, 1240; (j) H. Egami, R. Shimizu, Y. Usui and M. Sodeoka, J. Fluorine Chem., 2014, 167, 172; $(k)$ Y. Yasu, Y. Arai, R. Tomita, T. Koike and M. Akita, Org. Lett., 2014, 16, 780; (l) R. Tomita, Y. Yasu, T. Koike and M. Akita, Angew. Chem., Int. Ed., 2014, 53, 7144; $(m)$ R. Zhu and S. L. Buchwald, J. Am. Chem. Soc., 2015, 137, 8069; (n) Q.-H. Deng, J.-R. Chen, Q. Wei, Q.-Q. Zhao, L.-Q. Lu and W.-J. Xiao, Chem. Commun., 2015, 51, 3537; (o) N. Noto, K. Miyazawa, T. Koike and M. Akita, Org. Lett., 2015, 17, 3710; (p) Q. Wei, J.-R. Chen, X. Q. Hu, X. C. Yang, B. Lu and W. J. Xiao, Org. Lett., 2015, 17, 4464; (q) S. Jana, A. Ashokan, S. Kumar, A. Verma and S. Kumar, Org. Biomol. Chem., 2015, 13, 8411; (r) Y. Yang, Y. Liu, Y. Jiang, Y. Zhang and D. A. Vicic, J. Org. Chem., 2015, 80, 6639; $(s)$ L. Jarrige, A. Carboni, G. Dagousset, G. Levitre, E. Magnier and G. Masson, Org. Lett., 2016, 18, 2906; $(t)$ Y. Wu, G. Lu, T. Yuan, Z. Xu, L. Wan and C. Cai, Chem. Commun., 2016, 52, 13668; $(u)$ X. Bai, L. Lv and Z. Li, Org. Chem. Front., 2016, 3, 804; (v) N. Noto, T. Koike and M. Akita, J. Org. Chem., 2016, 81, 7064; (w) H. Y. Zhang, C. Ge, J. Zhao and Y. Zhang, Org. Lett., 2017, 19, 5260; (x) Y. F. Cheng,
X. Y. Dong, Q. S. Gu, Z. L. Yu and X.-Y. Liu, Angew. Chem., Int. Ed., 2017, 56, 8883.

4 Selected reports on transition-metal-catalysed aminotrifluoromethylations of styrenes: (a) Y. Yasu, T. Koike and M. Akita, Org. Lett., 2013, 15, 2136; (b) F. Wang, X. Qi, Z. Liang, P. Chen and G. Liu, Angew. Chem., Int. Ed., 2014, 53, 1881; (c) G. Dagousset, A. Carboni, E. Magnier and G. Masson, Org. Lett., 2014, 16, 4340; (d) J.-S. Lin, X.-Y. Dong, T.-T. Li, N.-C. Jiang, B. Tan and X.-Y. Liu, J. Am. Chem. Soc., 2016, 138, 9357; (e) L.-Z. Yu, Y. Wei and M. Shi, Chem. Commun., 2016, 52, 13163; $(f)$ K. Shen and Q. Wang, Org. Chem. Front., 2016, 3, 222; (g) H.-Y. Zhang, W. Huo, C. Ge, J. Zhao and Y. Zhang, Synlett, 2017, 28, 962.

5 Metal-free oxy-trifluoromethylations: (a) K. Uneyama, S. Watanabe, Y. Tokunaga, K. Kitagawa and Y. Sato, Bull. Chem. Soc. Jpn., 1992, 65, 1976; (b) Y. Sato, S. Watanabe and K. Uneyama, Bull. Chem. Soc. Jpn., 1993, 66, 1840; (c) C.-P. Zhang, Z.-L. Wang, Q.-Y. Chen, C.-T. Zhang, Y.-C. Gu and J.-C. Xiao, Chem. Commun., 2011, 47, 6632; (d) Y. Li and A. Studer, Angew. Chem., Int. Ed., 2012, 51, 8221; (e) H.-Q. Luo, Z.-P. Zhang, W. Dong and X.-Z. Luo, Synlett, 2014, 25, 1307; (f) Q. Lu, C. Liu, Z. Huang, Y. Ma, J. Zhang and A. Lei, Chem. Commun., 2014, 50, 14101; (g) J. Yu, H. Yang and H. Fu, Adv. Synth. Catal., 2014, 356, 3669; $(h)$ C. Liu, Q. Lu, Z. Huang, J. Zhang, F. Liao, P. Peng and A. Lei, Org. Lett., 2015, 17, 6034; (i) L. Huang, S.-C. Zheng, B. Tan and X.-Y. Liu, Org. Lett., 2015, 17, 1589; (j) M. Hartmann, Y. Li and A. Studer, Org. Biomol. Chem., 2016, 14, 206; (k) N.-Y. Yang, Z.-L. Li, L. Ye, B. Tan and X.-Y. Liu, Chem. Commun., 2016, 52, 9052; (l) J.-S. Lin, F.-L. Wang, X.-Y. Dong, W.-W. He, Y. Yuan, S. Chen and X.-Y. Liu, Nat. Commun., 2017, 8, 14841; (m) X.-T. Li, Q.-S. Gu, X.-Y. Dong, X. Meng and X.-Y. Liu, Angew. Chem., Int. Ed., 2018, 57, 7668.

6 Li's group reported a dihydrofuran-forming oxytrifluoromethylation using 1,3-diaryl diketone-containing alkene in the presence of Cu-catalyst; they proposed a radical cyclization mechanism involving $\mathrm{C}-\mathrm{O}$ bond formation between the radical carbon centre and $\mathrm{O}=\mathrm{C}$ group: X. Bai, L. Lv and Z. Li, Org. Chem. Front., 2016, 3, 804.

7 Nagano reported a metal-free hydroxy-trifluoromethylation reaction using $\mathrm{CF}_{3} \mathrm{I}$. This reaction involved hydrolysis of the iodo-trifluoromethylation intermediate, and thus can be classified as iodo-trifluoromethylation: (a) T. Yajima, C. Saito and H. Nagano, Tetrahedron, 2005, 61, 10203. Our group also reported KI-promoted, metal-free oxazolineforming trifluoromethylation of allylamides with Togni reagent, in which an iodo-trifluoromethylation intermediate is also involved: (b) S. Kawamura, D. Sekine and M. Sodeoka, J. Fluorine Chem., 2017, 203, 115.

8 (a) K. Arai, K. Watts and T. Wirth, ChemistryOpen, 2014, 3, 23; (b) N. Noto, T. Koike and M. Akita, Chem. Sci., 2017, 8, 6375. 9 (a) S. Kawamura and M. Sodeoka, Angew. Chem., Int. Ed., 2016, 55, 8740; (b) S. Kawamura, K. Dosei, E. Valverde, K. Ushida and M. Sodeoka, J. Org. Chem., 2017, 82, 12539.

10 Zard reported thio-trifluoromethylation of alkenes by using $S$-trifluoromethyl xanthates prepared from TFAA: 
F. Bertrand, V. Pevere, B. Quiclet-Sire and S. Z. Zard, Org. Lett., 2001, 3, 1069.

11 Trifluoromethylations of aromatic compounds with bis(trifluoroacetyl) peroxide (BTFAP): (a) M. Yoshida, T. Yoshida, M. Kobayashi and N. Kamigata, J. Chem. Soc., Perkin Trans. 1, 1989, 909; (b) H. Sawada, M. Nakayama, M. Yoshida, T. Yoshida and N. Kamigata, J. Fluorine Chem., 1990, 46, 423; (c) M. Matsui, S. Kawamura, K. Shibata, M. Mitani, H. Sawada and M. Nakayama, J. Fluorine Chem., 1992, 57, 209; (d) M. Nishida, S. Fujii, H. Kimoto, Y. Hayakawa, H. Sawada and L. A. Cohen, J. Fluorine Chem., 1993, 63, 43; (e) M. Matsui, S. Kondoh, K. Shibata and H. Muramatsu, Bull. Chem. Soc. Jpn., 1995, 68, 1042; (f) Y. Hayakawa, N. Terasawa and H. Sawada, Polymer, 2001, 42, 4081; $(g)$ S. Zhong, A. Hafner, C. Hussal, M. Nieger and S. Bräse, RSC Adv., 2015, 5, 6255.

12 Stephenson and co-workers reported the photochemical perfluoroalkylation with pyridine $N$-oxides/TFAA adduct:

(a) J. W. Beatty, J. J. Douglas, K. P. Cole and C. R. J. Stephenson, Nat. Commun., 2015, 6, 7919; (b) J. W. Beatty, J. J. Douglas, R. Miller, R. C. McAtee, K. P. Cole and C. R. J. Stephenson, Chem, 2016, 1, 456.

13 See ESI $\dagger$ for details.

14 Isolated yields were slightly lower than NMR yields because of hydrolysis of 2 during isolation by means of silica-gel column chromatography.
15 Methoxy group-containing substrate showed very high reactivity, and only the moderate yield of the desired product was obtained due to undesired side reactions, even if the reaction was carried out at $0{ }^{\circ} \mathrm{C}$.

16 (a) H. Egami, S. Kawamura, A. Miyazaki and M. Sodeoka, Angew. Chem., Int. Ed., 2013, 52, 7841; (b) S. Kawamura, H. Egami and M. Sodeoka, J. Am. Chem. Soc., 2015, 137, 4865.

17 The reaction of $N$-(2-vinylphenethyl)-p-toluenesulfonamide was examined. However, only $9 \%$ yield of tetrahydroisoquinoline was formed and $81 \%$ yield of an oxy-trifluoromethylation product (alcohol) was obtained. See ESI† for details.

18 Liu reported $\mathrm{Cu}$-catalyzed amino-trifluoromethylation of the same substrate with Togni reagent and obtained $\mathbf{4 b}$ with synselectivity: Y. Wang, M. Jiang and J.-T. Liu, Adv. Synth. Catal., 2016, 358, 1322.

19 V. D. Vuković, E. Richmond, E. Wolf and J. Moran, Angew. Chem., Int. Ed., 2017, 56, 3085.

20 M. Rubin and V. Gevorgyan, Org. Lett., 2001, 3, 2705.

21 As reported in ref. $9 a$, reaction of a simple alkene under the similar reaction conditions was very slow giving a small amount of complex mixture of products. A trace amount of $\mathrm{CF}_{3}$ radical generated via thermal decomposition could cause undesired reactions in the absence of an appropriate electron acceptor. 